\title{
A Geometric Morphometric Analysis of the Crown Form of the Maxillary Central Incisor in Humans
}

\author{
Akiko Kato'* , Makiko Kouchi², Masaaki Mochimaru², Ayamoto Isomura ${ }^{1}$, and Norikazu Ohno ${ }^{1}$ \\ ${ }^{1}$ Department of Oral Anatomy, School of Dentistry, Aichi-Gakuin University, 1-100 Kusumoto-cho, Chikusa-ku, \\ Nagoya 464-8650, Japan \\ ${ }^{2}$ Digital Human Research Center, National Institute of Advanced Industrial Science and Technology, 2-41-6 Aomi, \\ Koto-ku, Tokyo 135-0064, Japan
}

ABSTRACT Dental traits have been studied over a long period and grossly evaluated using standard reference plaques. However, grading by subjective observation may result in inter-observer measurement errors. We aimed to analyze crown models three-dimensionally to assess the morphology of the lingual surface termed shovel shape. Micro-CT scanned data of 38 maxillary central incisors stored at two different laboratories were used to create crown models of the outer enamel surface (OES) and the dentinoenamel junction (DEJ). Original crown data were evaluated according to the grade of shoveling into weak and strong groups. Homologous models consisting of the same number of data points

Studies of tooth crown morphology are essential in human biology and phylogeny. In particular, dental morphological traits have been recognized for their importance as a phenotypic expression of genetic differences between groups (Ohno, 1986; Irish, 1998). Traditional standard morphological analysis for comparing dental traits is performed by visual morphological observations (Hanihara, 1954, 1955; Suzuki and Sakai, 1966; Mizoguchi, 1977, 1978), which has a long history of making significant contributions to dental science. The approach is generally based on quantifying the relative expressions and frequencies of discrete traits by a standard reference plaque.

On the other hand, it has been reported that grading by standardized plaque is susceptible to inter- and intra-observer measurement errors (Mizoguchi, 1977; Turner and Hanihara, 1977; Nichol and Turner II, 1986; Haydenblit, 1996). Haydenblit (1996) reported that the percentage of intra-observer error greater than a onegrade scoring difference was $5.4 \%$ for 20 maxillary dental traits and $4.7 \%$ for 20 mandibular dental traits. Additionally, inter-observer error in the $>1$-grade variant-scoring percentage for a total of 47 traits ranged from $0.0 \%$ to $40.0 \%$. Mizoguchi (1978) estimated percent discordances between duplicated observations on the same sample; he reported a value of $10.7 \%$ for shoveling were created and the distance matrices between tooth models of OES and DEJ were respectively analyzed by using multidimensional scaling analysis (MDS) and principal component analysis. Student's t-test was used to compare corresponding scores between the two groups based on shovel-shape. The results of a t-test in the OES model indicated significant differences between the two groups. In contrast, the result in the DEJ model did not reveal a statistically significant difference. Our results indicate that geometric morphometric analysis of microCT scanned tooth crowns represents a powerful solution for the objective shape assessment of human teeth. Dental Anthropology 2011;24(1):1-10.

in the central incisor, though he emphasized that discordances are negligible in most cases unless there is obvious misjudgement. Although the difficulty associated with discrimination among tooth crown grades depends on type of tooth character and degree of expression, in some instances observational estimation can influence the consequential outcome. Therefore, objective approaches to classify tooth crown characteristics are desirable.

The aims of the present study were (1) to explore the differences between subjectively discriminated grading with standard plaque and objectively distinguished grading with geometric morphometrics, and (2) to determine whether the outer enamel surface (OES) or dentinoenamel junction (DEJ) form in tooth crown distinguishes the existence of shovel shape among a variety of maxillary central incisor morphologies. From the perspective of dental anthropology, tooth crown morphology is consequential for taxonomy as the grouping variable. As a preliminary investigation, we

*Correspondence to: Akiko Kato, Department of Oral Anatomy, School of Dentistry, Aichi-Gakuin University, 1-100 Kusumoto-cho, Chikusa-ku, Nagoya 464-8650, Japan

Tel: +81-52-751-2561; Fax: +81-52-752-5988

E-mail: a-kato@dpc.aichi-gakuin.ac.jp 
focused on shovel shape as the tooth crown character, which was first described by Hrdlička (1920). We adopted the Arizona State University (ASU) Dental Anthropology System (Turner et al., 1991) for visual discrimination. This is a frequently-used standard for evaluating dental traits (Irish, 1998; Irish and Guatelli-Steinberg, 2003; Manabe et al., 2003, 2008; Nwe Aung et al., 2005; Suzuki, 2005; Sasaki et al., 2005). We also performed morphometric analysis with three-dimensional (3D) tooth data from various populations for objective discrimination.

For objective evaluation of $3 \mathrm{D}$ data, the database of anthropometry of human data is globally available and can be commercially applied (Kouchi and Mochimaru, 2004, 2010; Mochimaru and Kouchi, 2000). Unlike the 2D data, 3D data have significance in detecting group average form. A homologous modeling method was developed to classify 3D body forms (Mochimaru et al., 1999; Mochimaru and Kouchi, 2000; Kouchi and Mochimaru, 2006), which is applied for designing products that fit to human body shape. The basic modeling technology based on subdivisions of the surface has been developed and applied to foot shape and body shape (Mochimaru et al., 1999; Kouchi and Mochimaru, 2010). This method allows computation of the average and variability of 3D body shapes in a sample. In the present study, we applied homologous modeling and compared the tooth crown shape related to OES and DEJ. A homologous model can represent the tooth as shape data with the same number of data points of the same topology. Finally, we discuss the differences between the results of grading with an ordinal-scale plaque and those obtained from morphometric analysis, exploring the possibility of geometric morphometrics to evaluate 3D tooth crown shape.

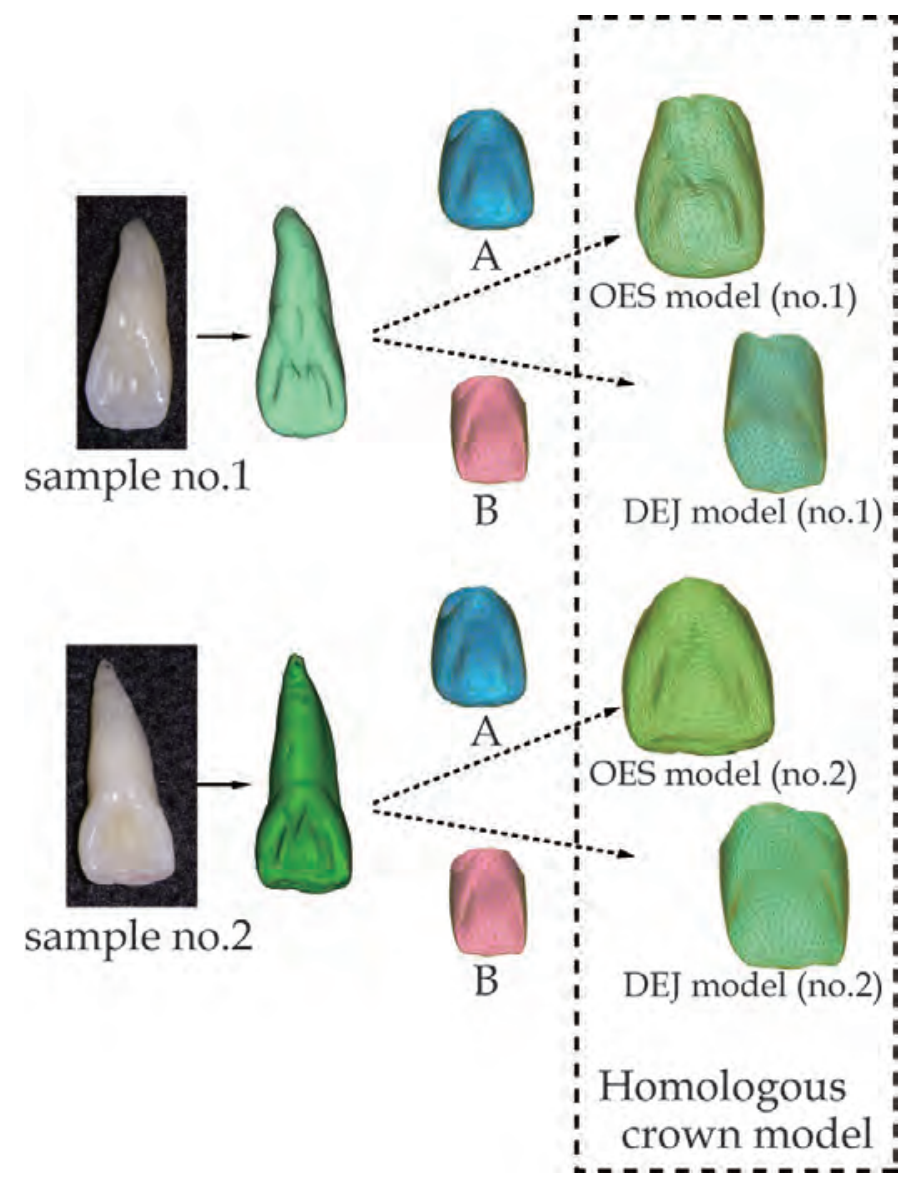

Fig. 1. Diagram of homologous crown model creation. Lingual surfaces of two examples (sample no. 1 and 2 are shown). Template crown models of the OES (A) and the DEJ (B) were used to construct homologous models.

TABLE 1. Maxillary central incisor data used in this study

\begin{tabular}{lccccc}
\hline Ethnic Group & $\mathrm{n}^{\mathrm{a}}$ & $\mathrm{WS}^{\mathrm{b}}$ & $\mathrm{SS}^{\mathrm{c}}$ & Data & Source \\
\hline Japanese & $23(22)$ & $9(12)$ & $14(10)$ & original & AGU \\
Indians & $2(2)$ & $2(2)$ & 0 & original & AGU \\
Burmese & $2(2)$ & $2(2)$ & 0 & original & AGU \\
Nepalese & $1(1)$ & $1(1)$ & 0 & original & AGU \\
Caucasian & $6(3)$ & $6(3)$ & 0 & CT & B\&H \\
Pacific-Islander & $1(0)$ & $1(0)$ & 0 & CT & B\&H \\
African-American & $3(2)$ & $3(2)$ & 0 & CT & B\&H \\
Totals & $38(32)$ & $24(22)$ & $14(10)$ & & \\
\hline
\end{tabular}

${ }^{a}$ n, sample size

${ }^{b}$ WS, weak shoveling (ASU: 0-2)

'SS, strong shoveling (ASU: 3-6)

${ }^{\mathrm{d}}$ AGU, Aichi-Gakuin University, Japan; B\&H, Brown and Herbranson Imaging, Inc., CA, USA

Numbers indicate dentinoenamel junction (DEJ) data

Numbers in parenthese show outer enamel surface (OES) data 


\section{MATERIALS AND METHODS}

\section{Study sample}

Micro-CT scanned data of 38 permanent upper left central incisors were obtained for geometric morphometric analysis of OES and DEJ of tooth crown. Table 1 lists the human populations from which the incisors used in this study were obtained. Japanese, Indians from India, Burmese and Nepalese incisors stored at Aichi-gakuin University (AGU) were scanned by micro-CT (SMX225CT, Shimadzu, Kyoto, Japan) at an isometric voxel resolution of 60 microns $(70 \mathrm{kV}, 50 \mu \mathrm{A}, 512 / 512$ matrix, 600 views, 360 degrees of rotation, 10 frame averaging). CT scan data of Caucasian, Pacific-Islander and AfricanAmerican teeth were provided by Brown and Herbranson Imaging, Inc. (B\&H, Palo Alto, CA, USA). The details of the $\mathrm{B} \& \mathrm{H}$ CT scan data were as follows: raw projection data of 16 bit, image size of 580/579/989 matrix and resolution of 20-60 microns isotropic cube.

Incisors were grouped into two classes based on the shoveling grade of the ASU Dental Anthropology System. Observations of the shovel shape for B\&H data were necessarily made from 3D models. As analyses based on the sum of shovel and semi-shovel grade were more appropriate to reduce the observational error (Suzuki and Sakai, 1966), the data were grouped into ASU grades 0-through-2 as the Weak Shoveling group (WS) and ASU grades 3-through- 6 as the Strong Shoveling group (SS). Six incisors that had enamel defects or caries were excluded from OES analysis in this study. The total numbers of teeth in WS and SS for OES analysis were 22 and 10, while those in WS and SS for DEJ analysis were 24 and 14, respectively. Right incisor data were mirror-imaged during 3D image reconstruction processing (described in the next section) in order to maximize the sample size. To reduce the size of the resulting files, image stacks were downsampled to 60 microns.

\section{Homologous Model Creation}

Figure 1 shows a diagram of homologous model creation. An image stack was imported into reconstruction software (VGStudio Max 2.0, Volume Graphics, GmbH, Heidelberg, Germany). During 3D image reconstruction, right incisor data were mirror-imaged to the left incisor. After calibration to define material and background, enamel and dentine tissues were segmented using
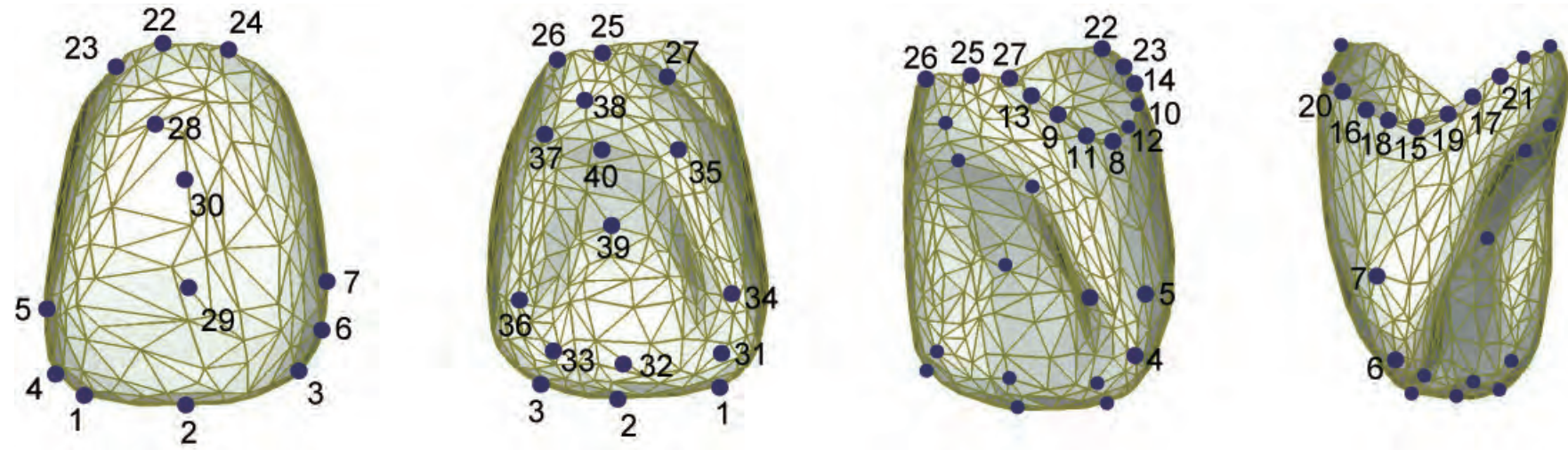

Fig. 2A. Depiction of the 40 landmarks used for OES surface model in this study.
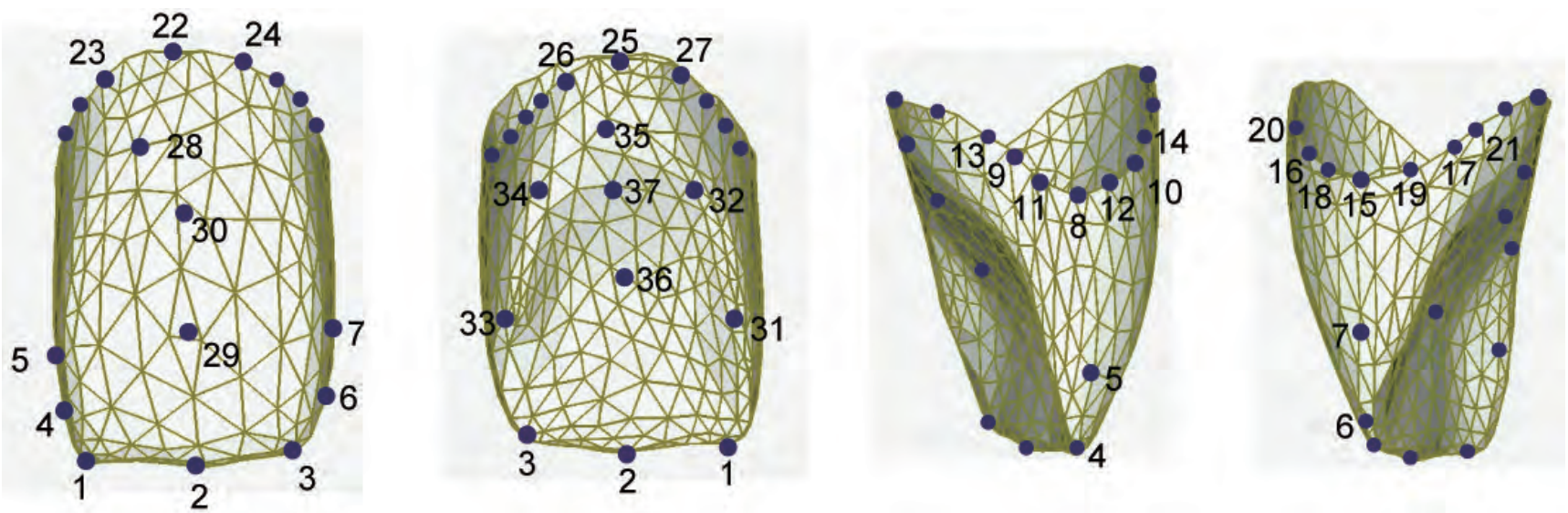

Fig. 2A. 40 landmarks used for OES surface model in this study. 
A
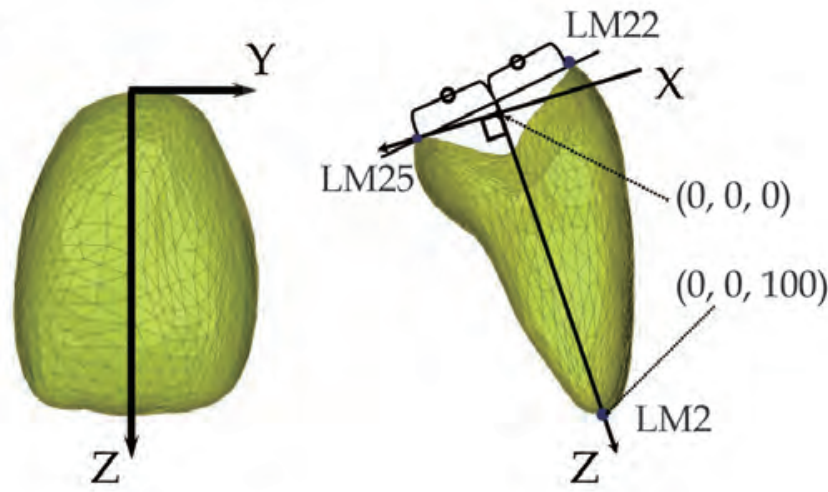

B
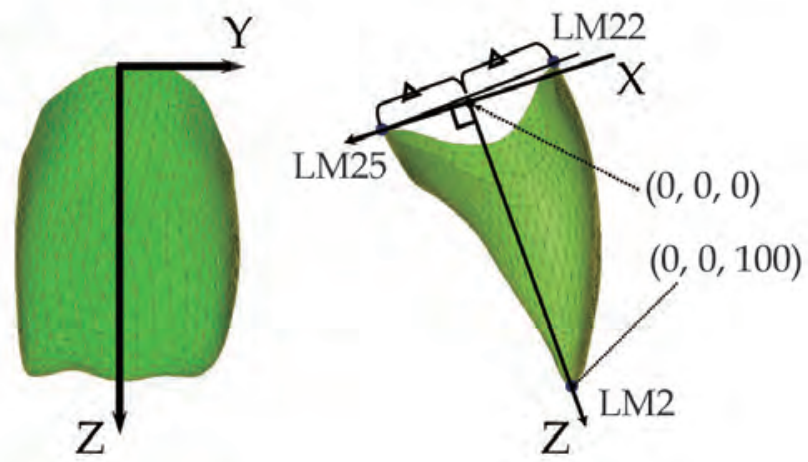

Fig. 3. Orientation of the OES model (A) and DEJ model (B). LM 2, LM 22, and LM 25 stand for the central point on the incisal edge, the center of the horizontal cross section of the highest point on the labial cervical line, and the highest point on the lingual cervical line, respectively.

the distribution of grayscale threshold values on its histogram, which arises from differences of mineralization of enamel and dentine. Then, the OES and DEJ form were respectively reconstructed as a triangulated surface model.

Before constructing a homologous model, we produced template crown models of the OES or the DEJ crown shape consisting of about 700 polygons using
Geomagic studio 9 (Geomagic, Inc., Durham, NC, USA). A template crown model exhibiting the shape of the enamel surface was produced from a segmented tooth crown enamel cap. In addition, the template crown model of the dentinoenamel junction shape was produced by substituting with the inner surface of the enamel cap model. That way, template models of the OES and DEJ were constructed and landmarks were applied on the model (Fig. 2A,B). 40 vertices for the OES model and 37 vertices for the DEJ model were manually assigned to anatomical landmarks. The template OES model had three more landmarks than the DEJ, and these were related to the thickness of the incisal edge. The template model automatically fits into the individual scanned point cloud of the maxillary central incisors by minimizing external and internal energy functions. The external energy function is based on the Euclidian distance between data points of the template model and those of the scanned data. The internal energy function is based on the local deformation of the template model. The vertices of the template model specified as landmarks were fit into the landmarks, and vertices generated by the subdivision surface were fit into measured point clouds with minimal deformation of the initial template model. As described above, the OES and DEJ homologous models were created for each sample by using Homologous Body Modeling software (HBM, Digital Human Technology Inc., Tokyo, Japan) and HBM-Rugle (Medic Engineering Corporation, Kyoto, Japan).

\section{Coordinate system}

Figure 3 illustrates the coordinate system used for crown model orientation. The Z-axis was the crown axis direction passing through the center of the horizontal cross-section of the highest point on the labial cervical line (landmark 22) and the highest point on the lingual cervical line (landmark 25), through the central point on the incisal edge (landmark 2). The X-axis was in the labiolingual direction passing the landmark 25 orthogonal

TABLE 2. Means, standard deviations and differences for measurements of Z- Y-X-directional length of polygon models obtained from CT scanned data

\begin{tabular}{|c|c|c|c|c|c|c|c|}
\hline & & $\begin{array}{c}\text { AGU } \\
\text { Mean (mm) }\end{array}$ & sd & $\begin{array}{c}\text { B\&H } \\
\text { Mean (mm) }\end{array}$ & $\mathrm{sd}$ & $\begin{array}{c}\text { Differences } \\
\text { AGU:B\&H (mm) }\end{array}$ & $\begin{array}{l}\text { Percentage } \\
\text { error }\end{array}$ \\
\hline \multirow[t]{3}{*}{ A } & Z & 22.13 & 0.8 & 22.58 & 0.9 & 0.45 & 2.0 \\
\hline & Y & 8.59 & 0.5 & 8.65 & 0.5 & 0.04 & 0.5 \\
\hline & $X$ & 6.86 & 0.2 & 7.23 & 0.6 & 0.38 & 5.5 \\
\hline \multicolumn{8}{|c|}{ B After Standardization } \\
\hline & $\mathrm{Z}$ & 20.00 & 0.0 & 20.00 & 0.0 & 0.00 & 0.0 \\
\hline & $\mathrm{Y}$ & 7.77 & 0.5 & 7.69 & 0.5 & 0.08 & 1.0 \\
\hline & $X$ & 6.20 & 0.3 & 6.42 & 0.7 & 0.22 & 3.5 \\
\hline
\end{tabular}

$\mathrm{n}=6$ 
TABLE 3. Significance probability (P value) from student ttest between MDS scores of WS group and those of SS group for all dimensions

\begin{tabular}{ccc}
\hline Dimension & OES $^{\mathrm{a}}$ & $\mathrm{DEJ}^{\mathrm{b}}$ \\
\hline 1 & 0.49 & 0.10 \\
2 & 0.27 & 0.14 \\
3 & 0.43 & 0.21 \\
4 & 0.44 & 0.77 \\
5 & $0.001^{* *}$ & 0.07 \\
\hline
\end{tabular}

${ }^{\mathrm{a}}$ outer enamel surface

${ }^{\mathrm{b}}$ dentinoenamel junction

$* * \mathrm{P}<0.01$

to the $\mathrm{Z}$-axis. The $\mathrm{Y}$-axis was in the mesiodistal direction. The origin $(0,0,0)$ was the intersection point of the $X$-axis and the Z-axis. Landmark 2 was made to lie at $(0,0,100)$ in each specimen in order to remove differences in crown height.

\section{Inter-System Comparison}

To assess the comparability of CT systems, we examined the possible measurement error of CT scanned data from two institutes. Six maxillary central incisors were scanned by both CT systems (AGU and B\&H). External surface models of the whole teeth were created by VGStudio Max 2.0. For inter-system comparison, the coordinate system used for crown model orientation was used and the length from central point on incisal edge to apical point of the root was scaled to be $20 \mathrm{~mm}$.

The size of polygon models was measured using the software. The shape errors between two polygon models were calculated.

\section{Statistical Analysis}

The distance between the two models was defined as the sum of the Euclidean distances between corresponding data points. The distance matrix between 32 models for OES and that between 38 models for DEJ was analyzed by the multidimensional scaling (MDS) method and principal component analysis (PCA). MDS is one of the factor analyses used to determine the spatial configuration of objects (Wickelmaier, 2003). MDS detects meaningful underlying dimensions that allow us to explain observed similarities or dissimilarities between objects. It generally attempts to arrange objects in a space with a particular number of dimensions, explaining the distance matrix in terms of fewer underlying dimensions to reduce the observed complexity of nature (Borg and Groenen, 2005; Bronstein et al., 2006). On the other hand, PCA, which is used in many studies (Stefan and Trinkaus, 1998; Hlusko and Mahaney, 2007; Bastier et al., 2008; Morimoto et al., 2008), summarizes data variation into fewer principal components corresponding to axes that account for the largest, second largest, and successively smaller proportion of the total sample variance. Kouchi and Mochimaru (2006) assessed the usefulness of PCA and MDS in analyzing variations in intra-individual shape change patterns and compared them. They reported that MDS is more efficient in information compression, so we assessed homologous models with MDS in addition to PCA to compare the information obtained from each analysis using Human Body Statistica (HBS, Digital Human Technologies Inc., Tokyo, Japan).

MDS and PCA scores in the WS and SS groups were compared by Student's t-test for OES or DEJ analyses. Statistical analysis was performed using statistical software (SPSS 15.0J for windows, SPSS Japan Inc., Tokyo, Japan). In order to interpret the obtained dimensions by MDS and PCA, virtual shapes with scores of \pm 3 S.D. for each of the three axes showing significant differences were calculated by using HBS (Mochimaru and Kouchi, 2000).

\section{RESULTS}

\section{Inter-System Comparison}

Six polygon models constructed by the authors from data acquired at AGU and B\&H were measured. Table 2A provides averages and standard deviations of tooth length, labiolingual diameter, and mesiodistal diameter substituted by the length along the $\mathrm{Z}$-axis, $\mathrm{X}$-axis, and

TABLE 4. Significance probability (P value) from student t-test between PCA scores of WS group and those of SS group for all PCS.

\begin{tabular}{cll}
\hline $\mathrm{PC}^{\mathrm{a}}$ & $\mathrm{OES}^{\mathrm{b}}$ & $\mathrm{DEJ}^{\mathrm{c}}$ \\
\hline 01 & 0.82 & 0.84 \\
02 & 0.22 & $0.02^{*}$ \\
03 & 0.55 & 0.58 \\
04 & 0.08 & 0.18 \\
05 & 0.09 & 0.25 \\
06 & 0.31 & 0.36 \\
07 & 0.21 & 0.72 \\
08 & $0.03^{*}$ & 0.17 \\
09 & 0.59 & 0.09 \\
10 & $0.01^{* *}$ & 0.06 \\
11 & 0.20 & 0.27 \\
12 & 0.74 & 0.54 \\
13 & 0.74 & 0.17 \\
14 & 0.71 & - \\
15 & 0.26 & - \\
16 & 0.67 & - \\
\hline
\end{tabular}

aprincipal component ${ }^{b}$ outer enamel surface 'dentinoenamel junction ${ }^{*} 0.05>\mathrm{P}>0.01$

$* * \mathrm{P}<0.01$ 


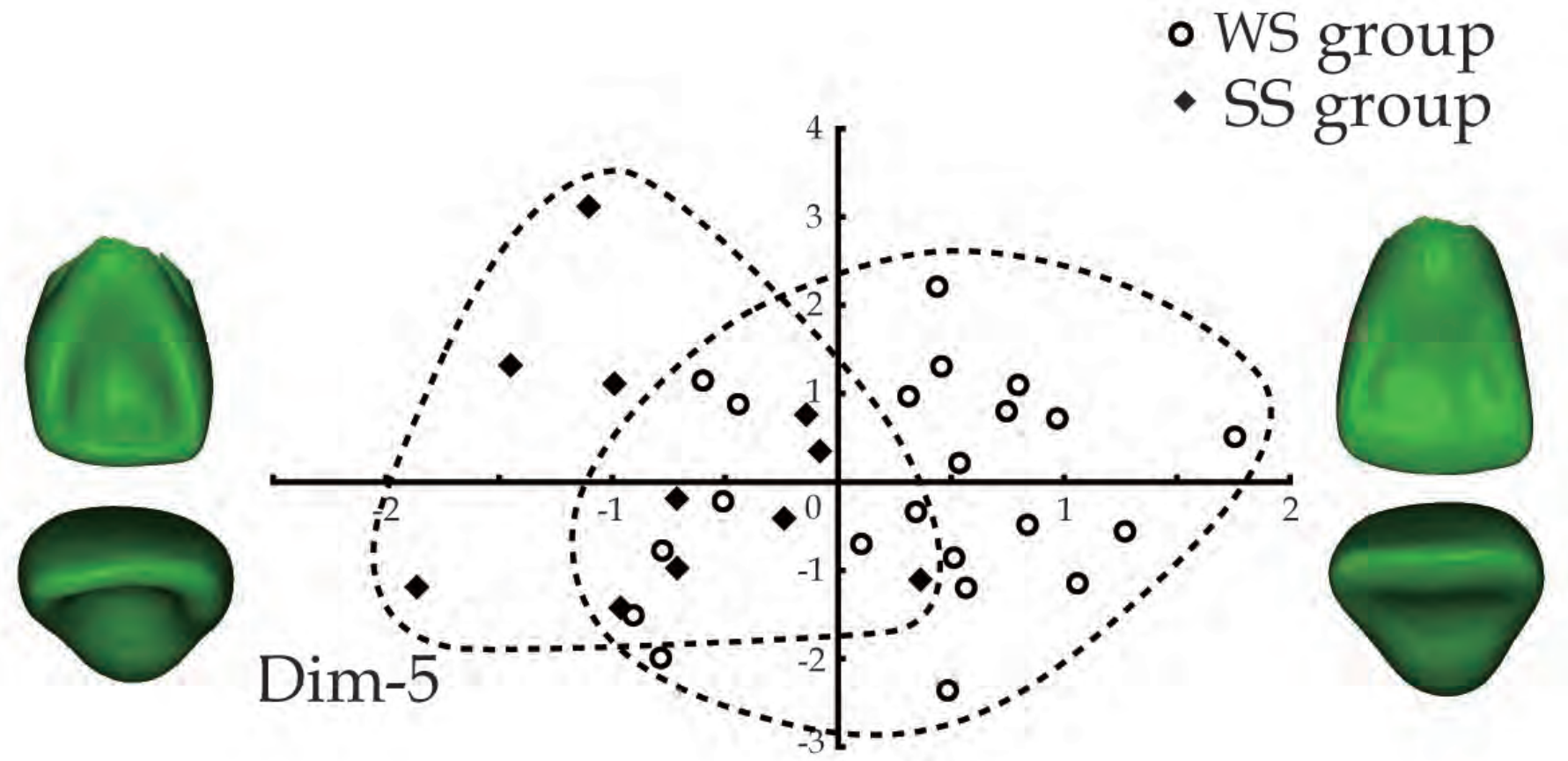

Fig. 4. Scatter plot of MDS scores in dimension-5 (Dim-5) for the OES model of WS and SS groups. +3 SD and -3 SD virtual shape models across dimension- 5 axis are indicated at the both end of the axis.

Y-axis, respectively. The percent errors between the two systems ranged from $0.5 \%$ to $5.5 \%$. Based on these results, the length of the present model was standardized. Table 2B shows the measured values after standardization. The percent errors between the two systems ranged from $0.0 \%$ to $3.5 \%$.

\section{MDS Scores}

A five-dimension solution was adopted for both the OES and DEJ analyses because $\mathrm{R}^{2}$ (squared correlation coefficient) was high (OES, 0.84; DEJ, 0.94). Table 3 shows the level of significance (P value) of the Student's t-test between MDS scores of the WS group and SS group for all dimensions. As MDS calculates spatial configuration of objects, the calculated factor of differences is called "dimension." The first dimension has the highest variance and each succeeding dimension in turn has the higher variance that is uncorrelated with the preceding dimension. Significant differences were found in only dimension-5 $(\mathrm{P}<0.01)$ between the WS and SS groups in the OES model. However, no significant difference in the DEJ model was observed.

Based on the calculated MDS score in dimension- 5 for the OES model, virtual shape models within -3 SD to +3 $\mathrm{SD}$ of the average were created. Figure 4 shows a scatter plot of MDS scores in dimension-5 for the OES model of the WS and SS groups. Virtual shape models with +3 SD and -3 SD across dimension- 5 axis are indicated at the ends of the axis. By observing the virtual shapes, MDS space can be interpreted over the axes. It was found that dimension- 5 related to thickness of the incisal edge and size of the mesial and distal marginal ridges in addition to the relative depth of the lingual surface.

\section{PCA Scores}

Almost $100 \%$ of total variance was explained by 16 PCs for the OES and 13 PCs for the DEJ. Table 4 shows the P values of the Student's t-test between PCA scores of the WS and SS group for all principal components. In PCA, significant differences in the OES model between the WS and SS groups were found in PC8 $(\mathrm{P}<0.05)$ and PC10 $(\mathrm{P}<0.01)$. The first $10 \mathrm{PCs}$ explain $82.3 \%$ of total variance. On the other hand, significant differences in DEJ were seen in PC2 ( $p$ 0.05). The first two PCs explain $41.9 \%$ of total variance. However, it is difficult to explain this component seen in the DEJ model. This is because the virtual shape expressed labiolingual thickness and mesiodistal length in addition to the depth of the lingual hollow. This means that the PC2 axis was not directly related to the shovel shape despite significant differences between PCA scores of the WS and SS groups. Thus, this component of DEJ model could be ignored.

Figure 5 shows a scatter plot of PCA scores in PC8 and PC10 for the OES model of the WS and SS groups. Virtual shape models within the +3 SD and -3 SD interval across each axis are indicated at the end of these axes. PC8 axis for the OES model was related to thickness of the incisal edge and size of the marginal ridge in parallel 


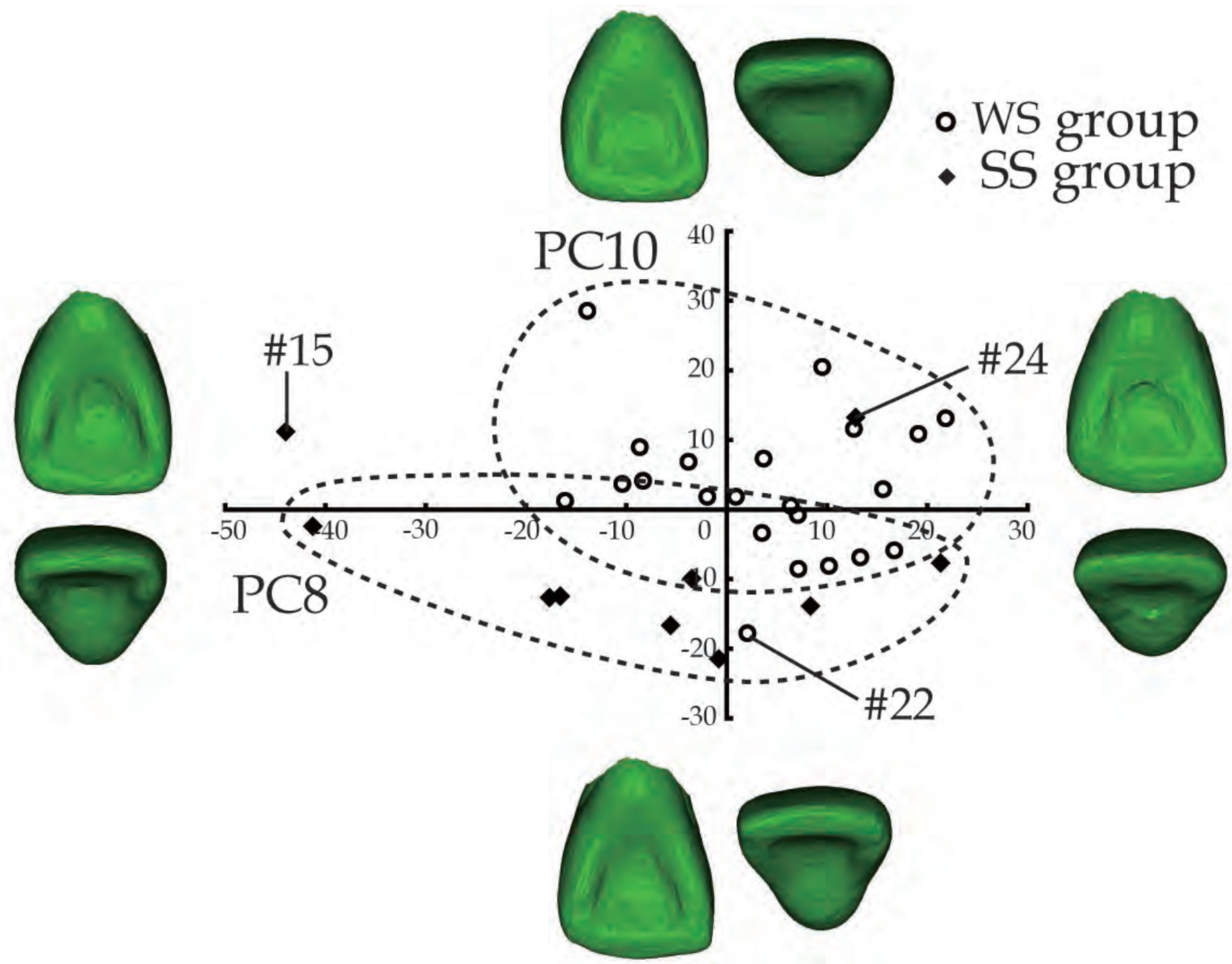

Fig. 5. Scatter plot of PCA scores in PC 8 and PC 10 for OES model of WS and SS groups. +3 SD and -3 SD virtual shape models across each axis are indicated at the end of these axes.

with shovel depth. PC10 for the OES model was related to the presence of the slope face (incline) following the lingual cingulum.

\section{DISCUSSION}

In the present study, the shoveling group subjectively discriminated with standard plaque corresponded to those groups objectively discriminated with 3D homologous crown models of outer enamel surface shape. In this section, we discuss some of the major issues and approaches involved in the acquisition of CT scanned data from different systems, and then we discuss the results of the present study.

Recent studies using micro-CT have contributed to our knowledge by evaluating a non-invasive method to analyze objects (Kono et al., 2002; Suwa and Kono, 2005; Smith and Tafforeau, 2008; Olejniczak et al., 2008).
Olejniczak and Grine (2006) compared physical sections to computer-generated micro-CT sections of recent primate teeth, and a difference of $3 \%$ to $5 \%$ was indicated between them. Their report revealed that measurement with the greatest care under proper conditions makes micro-CT useful. Furthermore, Olejniczak et al. (2007) compared different micro-CT systems to ensure that results are comparable and not machine-dependent and found that the measurements were comparable between systems (within 3\%).

In the present study, we analyzed human teeth, in which the degree of mineralization of enamel and dentine are similar among objects. Images acquired both at AGU and $\mathrm{B} \& \mathrm{H}$ appeared to be comparatively sharp and with few artifacts. Olejniczak and Grine (2006) reported that the ability of segmentation software to distinguish enamel and dentine differs in some cases from the ability of the 
human eye to detect the same two tissues. In general, data acquisition is performed by one operator under strictly controlled techniques. In addition, laboratory conditions are regulated to minimize inter-operator error. However, assessment of tooth morphology requires a large sample of certain taxa. Collections of required sample number may be limited in each laboratory. In particular, isolated teeth with a clear background are difficult to collect. Bailey et al. (2004) suggest that clear images with certain prescribed standards can be pooled together. In addition, data that are acquired under proper conditions, even if they were originally scanned for different purposes, can be gathered together and be analyzed as a large sample. In the present study, samples of different origin were combined and scanned under different conditions and techniques. To compensate for inter-system differences, segmentation differences used to distinguish enamel and dentine and the subsequent construction of homologous models were carried out by the first author. Nevertheless, an error ranging from $0.5 \%$ to $5.5 \%$ was revealed from the results of error verification in the present study. In order to compensate for these inter-system errors and account for differences in size, we standardized all the tooth length with the same value. As a result, shape data were standardized with acceptable accuracy $(0.0 \%$ to $3.5 \%)$. These results suggested that tooth models acquired from different systems could be used as valid data after some compensation. At the same time, it must be mentioned that this method is applied to a comparative study and not a measurement field. Collective data would benefit a wide range of researchers who engage in dental morphometric assessment (Kato and Ohno, 2008).

The expression of dental traits at the DEJ junction has been applied to extant and fossil hominoid taxa (Skinner et al., 2008, 2010). Statistically significant taxon-specific patterns at the DEJ that are not evident at the OES have also been reported (Skinner et al., 2009). Furthermore, if DEJ images can be obtained, the character retains its taxonomic value even in worn teeth. In the present study, we could not find any differences between the DEJ patterns of human teeth. This result was expected, since analysis for the OES shape should reflect the outcome of observations. Sakai and Hanamura (1973) describes the small component seen on the lingual surfaces of incisor as follows: "marginal ridges at the DEJ are less wide than at the OES, while thickness of marginal ridges is mostly the same". It is presumed that the morphometric differences found by examination of human teeth shape at the DEJ were too minor to be detected by the statistical analysis. For these reasons, we suggest that the present methodology using OES crown model could be appropriate for objective evaluation.

Shovel shape is characterized by "a peculiar, pronounced hollow of the lingual surface of the teeth, bounded laterally or surrounded by a well-defined elevated enamel border" (Hrdlička, 1920). Mizoguchi
(1978) reported that the correlation coefficients between the shoveling and marginal ridges are positive and considerably high, ranging from 0.55 to 0.82 , in the maxillary central incisor. In the present result of MDS analysis on the OES model, the dimension- 5 axis showed a shovel-shaped character. Virtual shape of +3 SD revealed thick incisal edge, and buccolingual midsection was also thick due to existence of the central ridge. Also, it showed narrower and thinner marginal ridges, and consequently a less deep hollow. On the other hand, virtual shape of -3 SD revealed thin incisal edge, and buccolingual midsection was not thick. Also, it showed wider and thicker marginal ridges, and consequently a deep hollow.

On the other hand, PCA resulted in the following PC8 axis: virtual shape with +3 SD showed that the marginal ridges are not prominent, the incisal edge is relatively thick, and consequently a less deep hollow. Virtual shape of -3 SD at the PC8 axis revealed that the marginal ridges are prominent, the incisal edge is not thick, and consequently a deep hollow. Furthermore, PCA resulted in the following PC10 axis: virtual shape of +3 SD showed a wide slope face from lingual cingulum, which was gradually flattening up to the lingual hollow. Virtual shape of -3 SD at PC10 revealed no slope from the lingual cingulum. Here, as the sample is not large enough, we cannot discuss the influences of mesial and distal marginal ridges with shovel shape. However, it is interesting for PCA analysis to extract a factor of the slope from lingual cingulum. As Mizoguchi (1978) reported, the central ridges decrease the extent of the shoveling. The present virtual shape showed weak shoveling with the slope face from the lingual cingulum and strong shoveling without the slope. This is interesting in terms of the small component on the lingual surface, which was related to the shoveling shape. Also, this component was extracted by PCA, not by MDS. That is, as Kouchi and Mochimaru (2006) suggested, MDS analysis is efficient for information compression. The present results revealed by MDS seemed to be the compressed shape factors compared to the results showed by PCA. Considering the differences between WS and SS groups combined with the result of calculated virtual forms of +3 SD and -3 SD here, these extracted results coincide with the characteristics of the shovel shape. Also, concerning the reason why the first few PCs do not exhibit significant differences between the WS and SS groups, it is attributed to the fact that the individual differences of crown form such as labiolingual width, mesiodistal length, and these mixed elements are relatively larger compared to differences of the shoveling.

As Figure 5 shows, there are three tooth crowns, \#24, $\# 22$ and \#15, that are apart from each group, and these outliers should be discussed here. First, \#24 was located in the area of the WS group, although it was classified as the SS group by observation, probably due to the 
existence of the incline. On the lingual surface of \#24, the lingual cingulum had a steep incline to the hollow of the incisal half area. Therefore, on observation, the focus was on the deep hollow and prominent lateral ridges and it was judged as having a strong shovel shape, whereas geometric analysis grouped \#24 into the WS group based on the presence of the slope. Second, \#22 was located in the area of the SS group, although it was classified as the WS group on observation. This may be due to the small pit and hollow above the lingual cingulum. It is considered that it was judged as having a weak shovel shape on observation due to less prominent lateral ridges. However, geometric analysis grouped \#22 in the strong shovel group based on the presence of the small pit and hollow above the lingual cingulum. Finally, in terms of \#15, we could see no apparent reason why it was apart from the group; it had a well-developed mesial marginal ridge and strong shoveling. In spite of the deep hollow in the lingual surface, \#15 was located in the upper area along with PC8 axis. Thus, all factors that influence the results are unknown at this time and require further investigation.

Objective evaluation of dental morphology has several advantages: 1) it does not involve man-made errors that accompany observation; 2) it enables analysis of data collected from around the world by 3D morphological data; and 3) it enables calculations of average tooth form in a group. There also are disadvantages, including 1) 3D morphological calculation could result in incompatible shape factor with conventional definition of dental traits; and 2) conditions in terms of landmark positioning or geometric algorithm of homologous model may affect the outcome of analysis. Nevertheless, potential contributions of these 3D morphometric data to dental science can be expected by overcoming these problems.

\section{CONCLUSION}

The study proposed geometric morphometric analysis of the maxillary central incisor crown form to assess degrees of lingual shoveling. The greatest merit of an objective methodology lies in the fact that a vast amount of data from all over the world could be analyzed all together. Although there are many technical challenges to overcome, we conclude that geometric morphometric analysis of micro-CT scanned tooth crowns represents a powerful solution for objective shape assessment of human teeth.

\section{ACKNOWLEDGEMENTS}

We are grateful to Paul Brown (Brown and Herbranson Imaging, Inc.) for providing CT scanned data. Tsuneo Iida contributed with software programming and image processing. We would like to thank also Masahito Natsuhara for assistance with micro-CT scanning, and Toyohisa Tanijiri for creating homologous models. This research was financially supported by the Hori Information Science Promotion Foundation and Aichi-Gakuin University.

\section{LITERATURE CITED}

Bailey SE, Pilbrow VC, Wood BA. 2004. Interobserver error involved in independent attempts to measure cusp base areas of Pan M1s. J Anat 205:323-331.

Bastir M, Rosas A, Lieberman DE, O'Higgins P. 2008. Middle cranial fossa anatomy and the origin of modern humans. Anat Rec 292:130-140.

Borg I, Groenen P. 2005. Modern multidimensional scaling: theory and applications. 2nd ed. New York: Springer.

Bronstein AM, Bronstein MM, Kimmel R. 2006. Generalized multidimensional scaling: a framework for isometry-invariant partial surface matching. Proc Natl Acad Sci 103:1168-1172.

Hanihara K. 1954. Studies on the deciduous dentition of the Japanese and the Japanese-American hybrids. I. Deciduous incisors. Anthropol Sci 63:168-185 (In Japanese).

Hanihara K. 1955. Studies on the deciduous dentition of the Japanese and the Japanese-American hybrids. II. Deciduous canines. Anthropol Sci 64:63-82 (In Japanese).

Haydenblit R. 1996. Dental variation among four prehispanic Mexican populations. Am J Phys Anthropol 100:225-246.

Hlusko LJ, Mahaney MC. 2007. A multivariate comparison of dental variation in wild and captive populations of baboons (Papio hamadryas). Arch Oral Biol 52:195200.

Hrdlička A. 1920. Shovel-shaped teeth. Am J Phys Anthropol 3:429-465.

Irish JD. 1998. Ancestral dental traits in recent SubSaharan Africans and the origins of modern humans. J Hum Evol 34:81-98.

Irish JD, Guatelli-Steinberg D. 2003. Ancient teeth and modern human origins: An expanded comparison of African Plio-Pleistocene and recent world dental samples. J Hum Evol 45:113-144.

Kato A, Ohno N. 2008. Construction of three-dimensional tooth model by micro-computed tomography and application for data sharing. Clin Oral Invest 13:43-46.

Kono RT, Suwa G, Tanijiri T. 2002. A three-dimensional analysis of enamel distribution patterns in human permanent first molars. Arch Oral Biol 47:867-875.

Kouchi M, Mochimaru M. 2004. Analysis of 3D face forms for proper sizing and CAD of spectacle frames. Ergonomics 47:1499-1516.

Kouchi M, Mochimaru M. 2006. Inter-individual variations in intra-individual shape change patterns. SAE Digital Human Modeling for Design and Engineering Conference 2006-01-2353.

Kouchi M, Mochimaru M. 2010. Simulation of the body 
shape after weight change for health-care services. In: Duffy VG, editor. Advances in Applied Digital Human Modeling. Indiana: CRC Press. P 217-222.

Manabe Y, Oyamada J, Kitagawa Y, Rokutanda A, Kato K, Matsushita T. 2003. Dental morphology of the Dawenkou Neolithic population in North China: implications for the origin and distribution of Sinodonty. J Hum Evol 45:369-380.

Manabe Y, Kitagawa Y, Oyamada J, Igawa K, Kato K, Kikuchi N, Maruo H, Kobayashi S, Rokutanda A. 2008. Population history of the northern and central Nansei Islands (Ryukyu island arc) based on dental morphological variations: geneflow from North Kyushyu to Nansei Islands. Anthropol Sci 116:49-65.

Mizoguchi Y. 1977. Genetic variability in tooth crown characters: Analysis by the tetrachoric correlation method. Bull Natl Sci Mus Ser D Anthropol 3:37-62.

Mizoguchi Y. 1978. Tooth crown characters on the lingual surfaces of the maxillary anterior teeth: Analysis of the correlations by the method of path coefficients. Bull Natl Sci Mus Ser D Anthropol 4:25-57.

Mochimaru M, Kouchi M, Dohi M. 1999. Analysis of foot forms using the FFD method and its application in grading shoe lasts. Ergonomics 43:1301-1313.

Mochimaru M, Kouchi M. 2000. Statistics for 3D human body forms. SAE Digital Human Modeling for Design and Engineering Conference 2000-01-2149..

Morimoto N, Ogihara N, Katayama K, Shiota K. 2008. Three-dimensional ontogenetic shape changed in the human cranium during the fetal period. J Anat 212:627-635.

Nichol CR, Turner CG II. 1986. Intra- and interobserver concordance in classifying dental morphology. Am J Phys Anthropol 69:299-315.

Nwe Aung N, Saruwatari L, Bo B, Sakai E, Manabe Y, Ohno N. 2005. Dental traits among five tribes in Myanmar (Burma). J Oral Biosci 47:272-279.

Ohno N. 1986. The inheritance of dental trait of a Hindu population in India. J Oral Biol 28:389-399 (In Japanese).

Olejniczak AJ, Grine FE. 2006. Assessment of the accuracy of dental enamel thickness measurements using microfocal X-ray computed tomography. Anat Rec 288A:263-275.

Olejniczak AJ, Tafforeau P, Smith TM, Temming H, Hublin JJ. 2007. Imaging systems for dental measurements. Am J Phys Anthropol 134:130-134.

Olejniczak AJ, Smith TM, Feeney RNM, Macchiarelly R, Mazurier A, Bondioli L, Rosas A, Fortea J, Rasilla M, Garcia-Tabernero A, Radovčić J, Skinner MM, Toussaint M, Hublin JJ. 2008. Dental tissue proportions and enamel thickness in Neandertal and modern human molars. J Hum Evol 55:12-23.
Sakai T, Hanamura H. 1973. A Morphological Study of Enamel-Dentin Border on the Japanese Dentition. VII. General Conclusion. Anthropol Sci 81:87-102 (In Japanese).

Sasaki K, Yamashita M, Ichikawa H, Ito R, Kobayashi S, Kanazawa E. 2005. Inter-population comparison of morphological traits in the upper canine. J Oral Sci 31:102-109 (In Japanese).

Skinner MM, Gunz P, Wood BA, Boesch C, Olejniczak AJ, Rosas A, Smith TM, Hublin JJ. 2008. Dental trait expression at the enamel-dentine junction of lower molars in extant and fossil hominoids. J Hum Evol 54:173-186.

Skinner MM, Wood BA, Hublin JJ. 2009. Protostylid expression at the enamel-dentine junction and enamel surface of mandibular molars of Paranthropus robustus and Australopithecus africanus. J Hum Evol 56:76-85.

Skinner MM, Evans A, Smith T, Jernvall J, Tafforeau P, Kupczik K, Olejniczak AJ, Rosas A, Radovčić J, Thackeray JF, Toussaint M, Hublin JJ. 2010. Brief communication: Contributions of enamel-dentine junction shape and enamel deposition to primate molar crown complexity. Am J Phys Anthropol 142:157-163.

Smith TM, Tafforeau P. 2008. New visions of dental tissue research: tooth development, chemistry, and structure. Evol Anthropol 17:213-226.

Stefan VH, Trinkaus E. 1998. Discrete trait and dental morphometric affinities of the Tabun 2 mandible. J Hum Evol 34:443-468.

Suwa G, Kono RT. 2005. A micro-CT based study of linear enamel thickness in the mesial cusp section of human molars: reevaluation of methodology and assessment of within-tooth, serial, and individual variation. Anthropol Sci 113:273-289.

Suzuki H. 2005. Dental characteristics in the Uigur Tribe of Xinjian-Uigur, China: Comparative analysis between Uigur and other populations. J Kyushu Dent Soc 59:61-79.

Suzuki M, Sakai T. 1966. Morphological analysis of the shovel-shaped teeth. Anthropol Sci 74:202-218 (In Japanese).

Turner CG II, Hanihara K. 1977. Additional features of Ainu dentition. V. Peopling of the pacific. Am J Phys Anthropol 46:13-24.

Turner CG II, Nichol CR, Scott GR. 1991. Scoring procedures for key morphological traits of the permanent dentition: The Arizona State University Dental Anthropology System. In: Kelley MA, Larsen CS, editors. Advances in dental anthropology. New York: Wiley-Liss. p 13-31.

Wickelmaier F. 2003. An Introduction to MDS. Denmark: Sound Quality Research Unit, Aalborg University. 